\title{
Fujita type theorem for a class of coupled quasilinear convection-diffusion equations
}

\author{
Yanan Zhou', Yan Leng ${ }^{2}$ and Yuanyuan $\mathrm{Nie}^{1 *}$ (D)
}

"Correspondence: nieyy@jlu.edu.cn

${ }^{1}$ School of Mathematics, Jilin

University, 130012 Changchun,

China

Full list of author information is available at the end of the article

\begin{abstract}
In this paper, we establish the Fujita type theorem for a homogeneous Neumann outer problem of the coupled quasilinear convection-diffusion equations and formulate the critical Fujita exponent. Besides, the influence of diffusion term, reaction term, and convection term on the global existence and the blow-up property of the problem is revealed. Finally, we discuss the large time behavior of the solution to the outer problem in the critical case and describe the asymptotic behavior of the solution.
\end{abstract}

MSC: 35B33; 35K20; 35K59

Keywords: Critical Fujita exponent; Fujita type theorem; Convection-diffusion equations

\section{Introduction}

In this paper, we consider the critical Fujita exponent of the following coupled quasilinear convection-diffusion equations:

$$
\begin{aligned}
& \frac{\partial u}{\partial t}=\Delta u^{m}+\kappa \frac{x}{|x|^{2}} \cdot \nabla u^{m}+|x|^{\lambda} v^{p}, \quad x \in \mathbb{R}^{n} \backslash \bar{B}_{1}, t>0, \\
& \frac{\partial v}{\partial t}=\Delta v^{m}+\kappa \frac{x}{|x|^{2}} \cdot \nabla v^{m}+|x|^{\mu} u^{q}, \quad x \in \mathbb{R}^{n} \backslash \bar{B}_{1}, t>0, \\
& \frac{\partial u^{m}}{\partial v}(x, t)=\frac{\partial v^{m}}{\partial v}(x, t)=0, \quad x \in \partial B_{1}, t>0, \\
& u(x, 0)=u_{0}(x), \quad v(x, 0)=v_{0}(x), \quad x \in \mathbb{R}^{n} \backslash \bar{B}_{1},
\end{aligned}
$$

where $p, q>m>1, \kappa \in \mathbb{R}, \lambda \geq 0, \mu=\frac{\lambda(q-m)+2(q-p)}{p-m} \geq 0$. In addition, $B_{1}$ denotes the unit ball in $\mathbb{R}^{n}, \boldsymbol{v}$ denotes the unit inner normal vector to $\partial B_{1}$, and $0 \leq u_{0}, v_{0} \in C_{0}\left(\mathbb{R}^{n}\right)$ are nontrivial.

In 1966, the first result of the exponent of the quasilinear diffusion equation was introduced by Fujita [5]. Precisely, he investigated the Cauchy problem of the semilinear equation

$$
\frac{\partial u}{\partial t}=\Delta u+u^{p}, \quad x \in \mathbb{R}^{n}, t>0,
$$

(c) The Author(s) 2021. This article is licensed under a Creative Commons Attribution 4.0 International License, which permits use, sharing, adaptation, distribution and reproduction in any medium or format, as long as you give appropriate credit to the original author(s) and the source, provide a link to the Creative Commons licence, and indicate if changes were made. The images or other third party material in this article are included in the article's Creative Commons licence, unless indicated otherwise in a credit line to the material. If material is not included in the article's Creative Commons licence and your intended use is not permitted by statutory regulation or exceeds the permitted use, you will need to obtain permission directly from the copyright holder. To view a copy of this licence, visit http://creativecommons.org/licenses/by/4.0/. 
and showed that the problem does not have any nontrivial global nonnegative solution if $1<p<p_{c}=1+2 / n$, whereas there exist both nontrivial global (with small initial data) and nonglobal nonnegative (with large initial data) solutions when $p>p_{c}=1+2 / n$. Among many other results, it proved $p=p_{c}$ belonged to the blow-up case by Hayakawa [12], Kobayashi et al. [13], and Weissler [27]. This is what we know as the blow-up theorem of Fujita type, and $p_{c}$ is called the critical Fujita exponent. Early results of the Fujita type theorem can be seen in the review articles of Deng [2], Levine [15], and relevant references. In recent years, there are a lot of Fujita's results, such as $[2,3,8,10,11,14,16-21,24-$ $26,30,31]$ and the references therein.

Among those works, Galaktionov et al. [6,7] considered the critical Fujita exponent of the Cauchy problem

$$
\frac{\partial u}{\partial t}=\Delta u^{m}+u^{p}, \quad x \in \mathbb{R}^{n}, t>0(p, m>1)
$$

and proved that the critical Fujita exponent is $p_{c}=m+2 / n$. Aguirre and Escobedo [1] demonstrated the Fujita type theorem of the following convective-diffusion equation:

$$
\frac{\partial u}{\partial t}=\Delta u^{m}+\boldsymbol{b}_{0} \cdot \nabla u^{q}+u^{p}, \quad x \in \mathbb{R}^{n}, t>0,
$$

where $q \geq 1, p>1, \boldsymbol{b}_{0} \in \mathbb{R}^{n}$. They demonstrated that the critical Fujita exponent was

$$
p_{c}=\min \left\{1+\frac{2}{n}, 1+\frac{2 q}{n+1}\right\} .
$$

Zheng and Wang [29] studied more general nonlinear convection-diffusion systems

$$
\begin{aligned}
& |x|^{\lambda_{1}} \frac{\partial u}{\partial t}=\Delta u^{m}+\kappa \frac{x}{|x|^{2}} \cdot \nabla u^{m}+|x|^{\lambda_{2}} u^{p}, \quad x \in \mathbb{R}^{n} \backslash \bar{\Omega}, t>0, \\
& \frac{\partial u^{m}}{\partial v}(x, t)=0, \quad x \in \partial \Omega, t>0, \\
& u(x, 0)=u_{0}(x), \quad x \in \mathbb{R}^{n} \backslash \bar{\Omega},
\end{aligned}
$$

where $p>m \geq 1, \kappa \in \mathbb{R},-2<\lambda_{1} \leq \lambda_{2}, \Omega$ is the bounded area in $\mathbb{R}^{n}$ with a smooth boundary $\partial \Omega$ and $B_{R_{1}} \subset \Omega \subset B_{R_{2}}$ for some $0<R_{1} \leq R_{2}$, and $B_{R}$ denotes the ball in $\mathbb{R}^{n}$ with radius $R$ and center at the origin, and $\boldsymbol{v}$ is a unit outer normal vector to $\partial B_{1}$. It displayed that the critical Fujita exponent is

$$
p_{c}= \begin{cases}m+\frac{2+\lambda_{2}}{n+\kappa+\lambda_{1}}, & \kappa>-n-\lambda_{1}, \\ +\infty, & \kappa \leq-n-\lambda_{1} .\end{cases}
$$

Following from a lot of results, it shows that critical Fujita exponent of a single equation is usually a constant, while the critical Fujita exponent of the coupled equations is usually a curve which is called the critical Fujita curve. In 1991, Escobedo and Herrero [4] investigated the following coupled systems:

$$
\frac{\partial u}{\partial t}=\Delta u+v^{p}, \quad \frac{\partial v}{\partial t}=\Delta v+u^{q}, \quad x \in \mathbb{R}^{n}, t>0
$$


where $p, q>0$, and showed that the Fujita curve is

$$
(p q)_{c}=1+\frac{2}{n} \max \{p+1, q+1\} .
$$

In [22], the authors studied the following Newtonian filtration system:

$$
\frac{\partial u}{\partial t}=\Delta u^{m}+v^{p}, \quad \frac{\partial v}{\partial t}=\Delta v^{m}+u^{q}, \quad x \in \mathbb{R}^{n}, t>0,
$$

where $0<m<1, p, q \geq 1$, and $p q>1$. It was proved that the critical Fujita curve is

$$
(p q)_{c}=m^{2}+\frac{2}{n} \max \{p+m, q+m\} .
$$

In [9], the authors studied the Fujita type theorem for the outer problem of the following coupled nonlinear diffusion equations with convective terms:

$$
\begin{array}{ll}
\frac{\partial u}{\partial t}=\Delta u+\kappa \frac{x}{|x|^{2}} \cdot \nabla u+|x|^{\lambda_{1}} v^{p}, & x \in \mathbb{R}^{n} \backslash \bar{B}_{1}, t>0, \\
\frac{\partial v}{\partial t}=\Delta v+\kappa \frac{x}{|x|^{2}} \cdot \nabla v+|x|^{\lambda_{2}} u^{q}, & x \in \mathbb{R}^{n} \backslash \bar{B}_{1}, t>0,
\end{array}
$$

and obtained

$$
(p q)_{c}= \begin{cases}1+\frac{\max \left\{p\left(2+\lambda_{2}\right)+\left(2+\lambda_{1}\right), q\left(2+\lambda_{1}\right)+\left(2+\lambda_{2}\right)\right\}}{n+\kappa}, & \kappa>-n \\ +\infty, & \kappa \leq-n .\end{cases}
$$

In this paper, we prove that the critical Fujita exponent is

$$
p_{c}= \begin{cases}m+\frac{\lambda+2}{n+\kappa}, & \kappa>-n, \\ +\infty, & \kappa \leq-n .\end{cases}
$$

The main attention of this paper is to prove the global existence and blow-up properties of solutions. For the global existence of the problem solution, we use the method of constructing the self-similar solution and the comparison principle to prove our conclusion. For the blow-up properties of solutions, we adopt the integral estimation method. It is noted that when discussing the global existence of solutions, we construct the self-similar upper solution to the system. In order to let the self-similar solutions have the same compact supported set, we introduce the perturbation term $(|x|+1)^{\mu}$. But the disturbance term has a negative impact on our results, which is the problem we need to solve.

The paper is organized as follows. In Sect. 2, we state some definitions and some theorems. Then, several useful auxiliary lemmas are given. In Sect. 4, we derive a Fujita type theorem for problem (1)-(4). At last, we study the asymptotic behavior of the solution to problem (1)-(4) in the critical case.

\section{Preliminaries}

In this section, we introduce the definition of the solutions to problem (1)-(4) that will be useful for the rest of the paper. 
Definition 2.1 Let $0<T \leq+\infty$. A pair of nonnegative functions $(u, v)$ is called a super (sub) solution to problem (1)-(4) in $(0, T)$ if

$$
u, v \in C\left([0, T), L_{\mathrm{loc}}^{m}\left(\mathbb{R}^{n}\right)\right) \cap L_{\mathrm{loc}}^{\infty}\left(0, T ; L^{\infty}\left(\mathbb{R}^{n}\right)\right),
$$

and the following integral inequalities

$$
\begin{aligned}
& \int_{0}^{T} \int_{\mathbb{R}^{n} \backslash B_{1}} u(x, t) \frac{\partial \varphi}{\partial t}(x, t) \mathrm{d} x \mathrm{~d} t+\int_{0}^{T} \int_{\mathbb{R}^{n} \backslash B_{1}} u^{m}(x, t) \Delta \varphi(x, t) \mathrm{d} x \mathrm{~d} t \\
& \quad-\kappa \int_{0}^{T} \int_{\mathbb{R}^{n} \backslash B_{1}} u^{m}(x, t) \operatorname{div}\left(\frac{1}{|x|^{2}} \varphi(x, t) x\right) \mathrm{d} x \mathrm{~d} t \\
& \quad+\int_{0}^{T} \int_{\mathbb{R}^{n} \backslash B_{1}}|x|^{\lambda} \nu^{p}(x, t) \varphi(x, t) \mathrm{d} x \mathrm{~d} t \\
& \quad-\int_{0}^{T} \int_{\partial B_{1}} u^{m}(x, t)\left(\frac{\partial \varphi}{\partial \boldsymbol{v}}(x, t)-\frac{\kappa}{|x|^{2}} \varphi(x, t) x \cdot v\right) \mathrm{d} \sigma \mathrm{d} t \\
& \quad+\int_{\mathbb{R}^{n} \backslash B_{1}} u_{0}(x) \varphi(x, 0) \mathrm{d} x \leq(\geq) 0, \\
& \int_{0}^{T} \int_{\mathbb{R}^{n} \backslash B_{1}} v(x, t) \frac{\partial \psi}{\partial t}(x, t) \mathrm{d} x \mathrm{~d} t+\int_{0}^{T} \int_{\mathbb{R}^{n} \backslash B_{1}} \nu^{m}(x, t) \Delta \psi(x, t) \mathrm{d} x \mathrm{~d} t \\
& \quad-\kappa \int_{0}^{T} \int_{\mathbb{R}^{n} \backslash B_{1}} v^{m}(x, t) \mathrm{div}\left(\frac{1}{|x|^{2}} \psi(x, t) x\right) \mathrm{d} x \mathrm{~d} t \\
& \quad+\int_{0}^{T} \int_{\mathbb{R}^{n} \backslash B_{1}}|x|^{\mu} u^{q}(x, t) \psi(x, t) \mathrm{d} x \mathrm{~d} t \\
& \quad-\int_{0}^{T} \int_{\partial B_{1}} v^{m}(x, t)\left(\frac{\partial \psi}{\partial \boldsymbol{v}}(x, t)-\frac{\kappa}{|x|^{2}} \psi(x, t) x \cdot v\right) \mathrm{d} \sigma \mathrm{d} t \\
& +\int_{\mathbb{R}^{n} \backslash B_{1}} v_{0}(x) \psi(x, 0) \mathrm{d} x \leq(\geq) 0
\end{aligned}
$$

are fulfilled for any $0 \leq \varphi, \psi \in C^{2,1}\left(\mathbb{R}^{n} \times[0, T)\right)$ vanishing when $t$ is near $T$ or $|x|$ is sufficiently large. $(u, v)$ is called a solution to problem $(1)-(4)$ in $(0, T)$ if it is both a supersolution and a subsolution.

Definition 2.2 A solution $(u, v)$ to problem (1)-(4) is said to blow up in a finite time $0<$ $T<+\infty$ if

$$
\|u(\cdot, t)\|_{L^{\infty}\left(\mathbb{R}^{n} \backslash B_{1}\right)}+\|v(\cdot, t)\|_{L^{\infty}\left(\mathbb{R}^{n} \backslash B_{1}\right)} \rightarrow+\infty \quad \text { as } t \rightarrow T^{-},
$$

which $T$ is called the blow-up time. Otherwise, $(u, v)$ is said to be global.

The following existence theorem and the comparison principle to problem (1)-(4) play an important role in proving our main results.

Theorem 2.1 (Local existence) When $0 \leq u_{0}, v_{0} \in L_{\text {loc }}^{1}\left(\mathbb{R}^{n}\right) \cap L^{\infty}\left(\mathbb{R}^{n}\right)$, the Cauchy problem (1)-(4) admits at least one solution locally in time. 
Theorem 2.2 (Comparison principle) For $0<T \leq+\infty$, assume that $\left(u^{*}, v^{*}\right)$ and $\left(u^{* *}, v^{* *}\right)$ are two solutions to system (1) and (2) with nonnegative initial data $u_{0}^{*}(x), v_{0}^{*}(x)$ and $u_{0}^{* *}(x)$, $v_{0}^{* *}(x)$ in $(0, T)$, respectively. If $\left(u_{0}^{*}(x), v_{0}^{*}(x)\right) \leq\left(u_{0}^{* *}(x), v_{0}^{* *}(x)\right)$ a.e. in $\mathbb{R}^{n}$, then $\left(u^{*}, v^{*}\right) \leq$ $\left(u^{* *}, v^{* *}\right)$ a.e. in $\mathbb{R}^{n} \times(0, T)$.

The proofs of Theorem 2.1 and Theorem 2.2 are the same as the one in $[23,25,28]$ and are omitted here.

\section{Auxiliary lemmas}

In order to research the blow-up property of solutions to problem (1)-(4), we need the following auxiliary lemmas.

Lemma 3.1 Assume that $(u, v)$ is a solution to problem (1)-(4). Then there exists $R_{0}>0$ depending only on $n$ and $\kappa$ such that, for any $l>R_{0}$,

$$
\begin{aligned}
& \frac{\mathrm{d}}{\mathrm{d} t} \int_{\mathbb{R}^{n} \backslash B_{1}} u(x, t) \psi_{l}(|x|) \mathrm{d} x \\
& \quad \geq-C_{0} l^{-2} \int_{B_{\delta l} \backslash B_{l}} u^{m}(x, t) \psi_{l}(|x|) \mathrm{d} x+\int_{\mathbb{R}^{n} \backslash B_{1}}|x|^{\lambda} v^{p}(x, t) \psi_{l}(|x|) \mathrm{d} x, \\
& \frac{\mathrm{d}}{\mathrm{d} t} \int_{\mathbb{R}^{n} \backslash B_{1}} v(x, t) \psi_{l}(|x|) \mathrm{d} x \\
& \quad \geq-C_{0} l^{-2} \int_{B_{\delta l} \backslash B_{l}} v^{m}(x, t) \psi_{l}(|x|) \mathrm{d} x+\int_{\mathbb{R}^{n} \backslash B_{1}}|x|^{\mu} u^{q}(x, t) \psi_{l}(|x|) \mathrm{d} x,
\end{aligned}
$$

where

$$
\delta=\left\{\begin{array}{ll}
2, & n+\kappa-1 \leq 0, \\
\frac{\pi}{n+\kappa-1}+1, & n+\kappa-1>0,
\end{array} \quad C_{0}=\frac{\pi^{2}}{(\delta-1)^{2}},\right.
$$

and

$$
\psi_{l}(r)= \begin{cases}r^{\kappa}, & 1 \leq r \leq l \\ \frac{1}{2} r^{\kappa}\left(1+\cos \frac{(r-l) \pi}{(\delta-1) l}\right), & l<r<\delta l \\ 0, & r \geq \delta l .\end{cases}
$$

Proof It follows from Definition 2.1 that

$$
\begin{aligned}
\frac{\mathrm{d}}{\mathrm{d} t} & \int_{\mathbb{R}^{n} \backslash B_{1}} u(x, t) \psi_{l}(|x|) \mathrm{d} x \\
= & \int_{B_{\delta l} \backslash B_{1}} u^{m}(x, t)\left(\Delta \psi_{l}(|x|)-\kappa \operatorname{div}\left(\frac{1}{|x|^{2}} \psi_{l}(|x|) x\right)\right) \mathrm{d} x \\
& \quad-\int_{\partial B_{1}} v^{m}(x, t)\left(\frac{\partial \psi_{l}(|x|)}{\partial v}-\frac{\kappa}{|x|^{2}} \psi_{l}(|x|) x \cdot v\right) \mathrm{d} \sigma+\int_{\mathbb{R}^{n} \backslash B_{1}} \nu^{p}(x, t) \psi_{l}(|x|) \mathrm{d} x \\
= & \int_{B_{\delta l} \backslash B_{1}} u^{m}(x, t)\left(\Delta \psi_{l}(|x|)-\kappa \operatorname{div}\left(\frac{1}{|x|^{2}} \psi_{l}(|x|) x\right)\right) \mathrm{d} x
\end{aligned}
$$




$$
+\int_{\mathbb{R}^{n} \backslash B_{1}} v^{p}(x, t) \psi_{l}(|x|) \mathrm{d} x, \quad t>0,
$$

where $\psi_{l}(r) \in C^{1}([0,+\infty))$ satisfies $\psi_{l}^{\prime}(0)=0$ and

$$
\frac{\partial \psi_{l}(|x|)}{\partial \boldsymbol{v}}-\frac{\kappa}{|x|^{2}} \psi_{l}(|x|) x \cdot \boldsymbol{v}=0, \quad x \in \partial B_{1} .
$$

For $0 \leq|x| \leq l$, it is easily verified that

$$
\begin{aligned}
& \Delta \psi_{l}(|x|)-\kappa \operatorname{div}\left(\frac{1}{|x|^{2}} \psi_{l}(|x|) x\right) \\
& =\psi_{l}^{\prime \prime}(|x|)+\frac{n-\kappa-1}{|x|} \psi_{l}^{\prime}(|x|)-\kappa \frac{n-2}{|x|^{2}} \psi_{l}(|x|)=0 .
\end{aligned}
$$

While for $l \leq|x| \leq \delta l$, a direct calculation gives

$$
\begin{aligned}
\Delta \psi_{l}(|x|)-\kappa \operatorname{div}\left(\frac{1}{|x|^{2}} \psi_{l}(|x|) x\right) \\
=-\frac{1}{2}(\delta-1)^{-1} \pi(n+\kappa-1) l^{-1}|x|^{\kappa-1} \sin \frac{(|x|-l) \pi}{(\delta-1) l} \\
\quad-\frac{1}{2}(\delta-1)^{-2} \pi^{2} l^{-2}|x|^{\kappa} \cos \frac{(|x|-l) \pi}{(\delta-1) l}
\end{aligned}
$$

If $n+\kappa-1 \leq 0$, one gets

$$
\begin{aligned}
\Delta \psi_{l}(|x|)-\kappa \operatorname{div}\left(\frac{1}{|x|^{2}} \psi_{l}(|x|) x\right) & \geq-\frac{1}{2}(\delta-1)^{-2} \pi^{2} l^{-2}|x|^{\kappa} \cos \frac{(|x|-l) \pi}{(\delta-1) l} \\
& \geq-\frac{1}{2}(\delta-1)^{-2} \pi^{2} l^{-2} \psi_{l}(|x|)
\end{aligned}
$$

If $n+\kappa-1>0$, we have

$$
\begin{aligned}
& \Delta \psi_{l}(|x|)-\kappa \operatorname{div}\left(\frac{1}{|x|^{2}} \psi_{l}(|x|) x\right) \\
& \geq-\frac{1}{2}(\delta-1)^{-2} \pi^{2} l^{-2}|x|^{\kappa} \sin \frac{(|x|-l) \pi}{(\delta-1) l}-\frac{1}{2}(\delta-1)^{-2} \pi^{2} l^{-2}|x|^{\kappa} \cos \frac{(|x|-l) \pi}{(\delta-1) l} \\
& \geq-\frac{1}{2}(\delta-1)^{-2} \pi^{2} l^{-2} \psi_{l}(|x|) .
\end{aligned}
$$

By (9)-(12), we obtain (7). Similarly, one can prove that (8) holds.

To prove the existence of a nontrivial global solution to problem (1)-(4), we introduce the following form of self-similar supersolutions to system (1) and (2):

$$
\begin{aligned}
& u(x, t)=(t+1)^{-\alpha} U\left((t+1)^{-\beta}|x|\right), \quad x \in \mathbb{R}^{n} \backslash B_{1}, t \geq 0, \\
& v(x, t)=(t+1)^{-\alpha} V\left((t+1)^{-\beta}|x|\right), \quad x \in \mathbb{R}^{n} \backslash B_{1}, t \geq 0,
\end{aligned}
$$

where

$$
\alpha=\frac{\lambda+2}{\lambda(m-1)+2(p-1)}, \quad \beta=\frac{(p-m) \alpha}{\lambda+2} .
$$


By a simple calculation, we show that

$$
\begin{aligned}
& \left(U^{m}\right)^{\prime \prime}(r)+\frac{n+\kappa-1}{r}\left(U^{m}\right)^{\prime}(r)+\beta r U^{\prime}(r)+\alpha U(r)+r^{\lambda} V^{p}(r) \leq 0, \\
& \left(V^{m}\right)^{\prime \prime}(r)+\frac{n+\kappa-1}{r}\left(V^{m}\right)^{\prime}(r)+\beta r V^{\prime}(r)+\alpha V(r)+r^{\mu} U^{q}(r) \leq 0,
\end{aligned}
$$

for any $r>0$. Then the self-similar function $(u, v)$ with the structure $(13)-(14)$ is a supersolution to (1) and (2).

Lemma 3.2 Assume that $m>1, \kappa>-n, p>p_{c}$ and set

$$
U(r)=V(r)=\left(\eta-A r^{2}\right)_{+}^{1 /(m-1)}, \quad r \geq 0,
$$

where $s_{+}=\max \{0, s\}, \eta>0$, and

$$
A=\frac{(m-1)(p-m) \alpha}{m(n+\kappa)\left(p+p_{c}-2 m\right)} .
$$

Then there exists sufficiently small $\eta>0$ such that (u,v) given by (13), (14), and (17) is a supersolution to system (1) and (2).

Proof It is clear that $U^{m}$ and $V^{m}$ satisfy (15) and (16) when $r \geq(\eta / A)^{1 / 2}$. For $0<r<$ $(\eta / A)^{1 / 2}$, a simple computation can obtain

$$
\begin{aligned}
& \left(U^{m}\right)^{\prime \prime}(r)+\frac{n+\kappa-1}{r}\left(U^{m}\right)^{\prime}(r)+\beta r U^{\prime}(r)+\alpha U(r) \\
& \quad=\left(\frac{2 A}{m-1}\left(\frac{2 A m}{m-1}-\beta\right) U^{1-m}(r)+\left(\alpha-\frac{2 A m(n+\kappa)}{m-1}\right)\right) U(r)
\end{aligned}
$$

and

$$
\begin{aligned}
& \left(V^{m}\right)^{\prime \prime}(r)+\frac{n+\kappa-1}{r}\left(V^{m}\right)^{\prime}(r)+\beta r V^{\prime}(r)+\alpha V(r) \\
& \quad=\left(\frac{2 A}{m-1}\left(\frac{2 A m}{m-1}-\beta\right) V^{1-m}(r)+\left(\alpha-\frac{2 A m(n+\kappa)}{m-1}\right)\right) V(r) .
\end{aligned}
$$

Due to $\frac{2 A m}{m-1}<\beta$, there exists sufficiently small $\eta_{1}>0$ such that, for $0<\eta<\eta_{1}$,

$$
\begin{aligned}
& \left(U^{m}\right)^{\prime \prime}(r)+\frac{n+\kappa-1}{r}\left(U^{m}\right)^{\prime}(r)+\beta r U^{\prime}(r)+\alpha U(r)<-\frac{\left(p-p_{c}\right) \alpha U(r)}{2\left(p+p_{c}-2 m\right)} \\
& \left(V^{m}\right)^{\prime \prime}(r)+\frac{n+\kappa-1}{r}\left(V^{m}\right)^{\prime}(r)+\beta r V^{\prime}(r)+\alpha V(r)<-\frac{\left(p-p_{c}\right) \alpha V(r)}{2\left(p+p_{c}-2 m\right)}
\end{aligned}
$$

Then, due to $\lambda, \mu>0$ and the definition of $U, V$, there exists $\eta_{2}>0$ such that, for any $0<\eta<\eta_{2}$,

$$
\begin{array}{ll}
r^{\mu} U^{q-1}(r) \leq A^{-\mu / 2} \eta^{(q-1) /(m-1)+\mu / 2}<\frac{\left(p-p_{c}\right) \alpha}{2\left(p+p_{c}-2 m\right)}, & 0<r<\left(\frac{\eta}{A}\right)^{1 / 2} \\
r^{\lambda} V^{p-1}(r) \leq A^{-\lambda / 2} \eta^{(p-1) /(m-1)+\lambda / 2}<\frac{\left(p-p_{c}\right) \alpha}{2\left(p+p_{c}-2 m\right)}, & 0<r<\left(\frac{\eta}{A}\right)^{1 / 2}
\end{array}
$$


Combining the above inequations with (18) and (19), we can see that for sufficiently small $0<\eta_{2}<\eta_{1}$ and $0<\eta<\eta_{2}<\eta_{1}$, one gets (15) and (16). Thus, (u,v) given by (13), (14), and (17) is a supersolution of system (1) and (2).

\section{Blow-up theorems of Fujita type}

In this section, we establish the blow-up theorems of Fujita type for problem (1)-(4). First, we consider the case $\kappa \leq-n$.

Theorem 4.1 Assume that $p, q>m, \lambda, \mu \geq 0, \kappa \leq-n$, and $0 \leq u_{0}, v_{0} \in C_{0}\left(\mathbb{R}^{n} \backslash B_{1}\right)$ are nontrivial, the solution to problem (1)-(4) blows up in a finite time.

Proof Let $(u, v)$ be the solution to problem (1)-(4). Denote

$$
w_{l}(t)=\int_{\mathbb{R}^{n} \backslash B_{1}}(u(x, t)+v(x, t)) \psi_{l}(|x|) \mathrm{d} x, \quad t \geq 0 .
$$

For any $l>R_{0}$, Lemma 3.1 shows that

$$
\begin{aligned}
\frac{\mathrm{d}}{\mathrm{d} t} w_{l}(t) \geq & -\frac{C_{0}}{l^{2}} \int_{B_{\delta l} \backslash B_{l}} u^{m}(x, t) \psi_{l}(|x|) \mathrm{d} x+\int_{\mathbb{R}^{n} \backslash B_{1}}|x|^{\mu} u^{q}(x, t) \psi_{l}(|x|) \mathrm{d} x \\
& -\frac{C_{0}}{l^{2}} \int_{B_{\delta l} \backslash B_{l}} v^{m}(x, t) \psi_{l}(|x|) \mathrm{d} x+\int_{\mathbb{R}^{n} \backslash B_{1}}|x|^{\lambda} \nu^{p}(x, t) \psi_{l}(|x|) \mathrm{d} x .
\end{aligned}
$$

The Hölder inequality leads to

$$
\begin{aligned}
& \int_{B_{\delta l} \backslash B_{l}} u^{m}(x, t) \psi_{l}(|x|) \mathrm{d} x \\
& \leq C_{1} l^{n+\kappa-m(n+\kappa+\mu) / q}\left(\int_{\mathbb{R}^{n} \backslash B_{1}}|x|^{\mu} u^{q}(x, t) \psi_{l}(|x|) \mathrm{d} x\right)^{m / q}, \\
& \int_{B_{\delta l} \backslash B_{l}} v^{m}(x, t) \psi_{l}(|x|) \mathrm{d} x \\
& \quad \leq C_{1} l^{n+\kappa-m(n+\kappa+\lambda) / p}\left(\int_{\mathbb{R}^{n} \backslash B_{1}}|x|^{\mu} \nu^{p}(x, t) \psi_{l}(|x|) \mathrm{d} x\right)^{m / p},
\end{aligned}
$$

where $C_{1}>0$ is a positive constant independent of $l$. Substituting (22) and (23) into (21) shows that

$$
\begin{aligned}
& \frac{\mathrm{d}}{\mathrm{d} t} w_{l}(t) \\
& \geq\left(\int_{\mathbb{R}^{n} \backslash B_{1}}|x|^{\mu} u^{q}(x, t) \psi_{l}(|x|) \mathrm{d} x\right)^{m / q}\left(\left(\int_{\mathbb{R}^{n} \backslash B_{1}}|x|^{\mu} u^{q}(x, t) \psi_{l}(|x|) \mathrm{d} x\right)^{(q-m) / q}\right. \\
& \left.\quad-C_{0} C_{1} l^{-2+n+\kappa-m(n+\kappa+\mu) / q}\right) \\
& \quad+\left(\int_{\mathbb{R}^{n} \backslash B_{1}}|x|^{\lambda} v^{p}(x, t) \psi_{l}(|x|) \mathrm{d} x\right)^{m / p}\left(\left(\int_{\mathbb{R}^{n} \backslash B_{1}}|x|^{\lambda} v^{p}(x, t) \psi_{l}(|x|) \mathrm{d} x\right)^{(p-m) / p}\right. \\
& \left.\quad-C_{0} C_{1} l^{-2+n+\kappa-m(n+\kappa+\lambda) / p}\right) .
\end{aligned}
$$


Owing to the Hölder inequality, for any $t>0$, we have

$$
\begin{aligned}
& \int_{\mathbb{R}^{n} \backslash B_{1}} u(x, t) \psi_{l}(|x|) \mathrm{d} x \\
& \leq\left(\int_{B_{\delta l} \backslash B_{1}}|x|^{-\mu /(q-1)} \psi_{l}(|x|) \mathrm{d} x\right)^{(q-1) / q}\left(\int_{\mathbb{R}^{n} \backslash B_{1}}|x|^{\mu} u^{q}(x, t) \psi_{l}(|x|) \mathrm{d} x\right)^{1 / q} \\
& \int_{\mathbb{R}^{n} \backslash B_{1}} v(x, t) \psi_{l}(|x|) \mathrm{d} x \\
& \leq\left(\int_{B_{\delta l} \backslash B_{1}}|x|^{-\lambda /(p-1)} \psi_{l}(|x|) \mathrm{d} x\right)^{(p-1) / p}\left(\int_{\mathbb{R}^{n} \backslash B_{1}}|x|^{\lambda} v^{p}(x, t) \psi_{l}(|x|) \mathrm{d} x\right)^{1 / p}
\end{aligned}
$$

which imply

$$
\begin{aligned}
& \int_{\mathbb{R}^{n} \backslash B_{1}}|x|^{\mu} u^{q}(x, t) \psi_{l}(|x|) \mathrm{d} x \\
& \geq \begin{cases}C_{2}\left(\int_{\mathbb{R}^{n} \backslash B_{1}} u(x, t) \psi_{l}(|x|) \mathrm{d} x\right)^{q} l^{n+\kappa+\mu-q(n+\kappa),} & \text { if } A(q, \mu)<0, \\
C_{2}\left(\int_{\mathbb{R}^{n} \backslash B_{1}} u(x, t) \psi_{l}(|x|) \mathrm{d} x\right)^{q}(\ln l)^{-(q-1)}, & \text { if } A(q, \mu)=0, \\
C_{2}\left(\int_{\mathbb{R}^{n} \backslash B_{1}} u(x, t) \psi_{l}(|x|) \mathrm{d} x\right)^{q}, & \text { if } A(q, \mu)>0,\end{cases} \\
& \int_{\mathbb{R}^{n} \backslash B_{1}}^{|x|^{\lambda} v^{p}(x, t) \psi_{l}(|x|) \mathrm{d} x} \\
& \geq \begin{cases}C_{2}\left(\int_{\mathbb{R}^{n} \backslash B_{1}} v(x, t) \psi_{l}(|x|) \mathrm{d} x\right)^{p} l^{n+\kappa+\lambda-p(n+\kappa),} & \text { if } A(p, \lambda)<0, \\
C_{2}\left(\int_{\mathbb{R}^{n} \backslash B_{1}} v(x, t) \psi_{l}(|x|) \mathrm{d} x\right)^{p}(\ln l)^{-(p-1)}, & \text { if } A(p, \lambda)=0, \\
C_{2}\left(\int_{\mathbb{R}^{n} \backslash B_{1}} v(x, t) \psi_{l}(|x|) \mathrm{d} x\right)^{p}, & \text { if } A(p, \lambda)>0,\end{cases}
\end{aligned}
$$

where $C_{2}>0$ is a positive constant independent of $l$ and $A(q, \mu)=n+\kappa+\mu-q(n+\kappa)$, $A(p, \lambda)=n+\kappa+\lambda-p(n+\kappa)$. Here, it should be pointed out that the above discussion only requires $p, q>m$.

Due to $\kappa \leq-n$, it is easy to verify that $A(q, \mu)>0, A(p, \lambda)>0$. From (24)-(26),

$$
\begin{aligned}
& \frac{\mathrm{d}}{\mathrm{d} t} w_{l}(t) \\
& \quad \geq C_{2}^{m / q}\left(\int_{\mathbb{R}^{n} \backslash B_{1}} u(x, t) \psi_{l}(|x|) \mathrm{d} x\right)^{m}\left(C_{2}^{(q-m) / q}\left(\int_{\mathbb{R}^{n} \backslash B_{1}} u(x, t) \psi_{l}(|x|) \mathrm{d} x\right)^{q-m}\right. \\
& \left.\quad-C_{0} C_{1} l^{-2+n+\kappa-m(n+\kappa+\mu) / q}\right) \\
& \quad+C_{2}^{m / p}\left(\int_{\mathbb{R}^{n} \backslash B_{1}} v(x, t) \psi_{l}(|x|) \mathrm{d} x\right)^{m}\left(C_{2}^{(p-m) / p}\left(\int_{\mathbb{R}^{n} \backslash B_{1}} v(x, t) \psi_{l}(|x|) \mathrm{d} x\right)^{p-m}\right. \\
& \left.\quad-C_{0} C_{1} l^{-2+n+\kappa-m(n+\kappa+\lambda) / p}\right) .
\end{aligned}
$$

For sufficiently large $l_{1}>1$, and note that $-2+n+\kappa-m(n+\kappa+\mu) / q<0,-2+n+\kappa-m(n+$ $\kappa+\lambda) / p<0$, one can get

$$
\begin{aligned}
& \frac{\mathrm{d}}{\mathrm{d} t} w_{l_{1}}(t) \\
& \quad \geq C_{2}^{m / q}\left(\int_{\mathbb{R}^{n} \backslash B_{1}} u(x, t) \psi_{l_{1}}(|x|) \mathrm{d} x\right)^{m}
\end{aligned}
$$




$$
\begin{aligned}
& \quad \times \frac{1}{2} C_{2}^{(q-m) / q}\left(\int_{\mathbb{R}^{n} \backslash B_{1}} u(x, t) \psi_{l_{1}}(|x|) \mathrm{d} x\right)^{q-m} \\
& +C_{2}^{m / p}\left(\int_{\mathbb{R}^{n} \backslash B_{1}} v(x, t) \psi_{l_{1}}(|x|) \mathrm{d} x\right)^{m} \\
& \quad \times \frac{1}{2} C_{2}^{(p-m) / p}\left(\int_{\mathbb{R}^{n} \backslash B_{1}} v(x, t) \psi_{l_{1}}(|x|) \mathrm{d} x\right)^{p-m} \\
& \geq C_{3}\left(\left(\int_{\mathbb{R}^{n} \backslash B_{1}} u(x, t) \psi_{l_{1}}(|x|) \mathrm{d} x\right)^{q}+\left(\int_{\mathbb{R}^{n} \backslash B_{1}} v(x, t) \psi_{l_{1}}(|x|) \mathrm{d} x\right)^{p}\right) \\
& \geq 2^{p+q} C_{3} \cdot \min \left\{w_{l_{1}}^{p}(t), w_{l_{1}}^{q}(t)\right\},
\end{aligned}
$$

where $C_{3}>0$ is a constant depending on $l_{1}$. Since $p, q>m>1$, there exists $0<T<+\infty$ such that

$$
w_{l_{1}}(t)=\int_{\mathbb{R}^{n} \backslash B_{1}}(u(x, t)+v(x, t)) \psi_{l_{1}}(|x|) \mathrm{d} x \rightarrow+\infty, \quad t \rightarrow T^{-} .
$$

Obviously, supp $\psi_{l_{1}}(x)=B_{2 l_{1}}$. Then one gets

$$
\|u(\cdot, t)\|_{L^{\infty}\left(\mathbb{R}^{n} \backslash B_{1}\right)}+\|v(\cdot, t)\|_{L^{\infty}\left(\mathbb{R}^{n} \backslash B_{1}\right)} \rightarrow+\infty, \quad t \rightarrow T^{-} .
$$

That is to say, $(u, v)$ blows up in a finite time.

Next, we discuss the case $\kappa>-n$.

Theorem 4.2 Assume that $p, q>m>1, \lambda, \mu>0, \kappa>-n$, and $0 \leq u_{0}, v_{0} \in C_{0}\left(\mathbb{R}^{n} \backslash B_{1}\right)$ are nontrivial. Then, for $p<p_{c}$, any nontrivial solution to problem (1)-(4) blows up in a finite time.

Proof Let $(u, v)$ be a nontrivial solution to problem (1)-(4). Set

$$
w_{l}(t)=\int_{\mathbb{R}^{n} \backslash B_{1}}\left(u(x, t)+l^{\theta} v(x, t)\right) \psi_{l}(|x|) \mathrm{d} x, \quad t \geq 0,
$$

where $\theta$ is a constant determined below. According to Lemma 3.1, for any $l>R_{0}$,

$$
\begin{aligned}
& \frac{\mathrm{d}}{\mathrm{d} t} w_{l}(t) \\
& \quad \geq-C_{0} l^{-2} \int_{B_{\delta l} \backslash B_{l}} u^{m}(x, t) \psi_{l}(|x|) \mathrm{d} x+l^{\theta} \int_{\mathbb{R}^{n} \backslash B_{1}}|x|^{\mu} u^{q}(x, t) \psi_{l}(|x|) \mathrm{d} x \\
& \quad-C_{0} l^{-2+\theta} \int_{B_{\delta l} \backslash B_{l}} v^{m}(x, t) \psi_{l}(|x|) \mathrm{d} x+\int_{\mathbb{R}^{n} \backslash B_{1}}|x|^{\lambda} v^{p}(x, t) \psi_{l}(|x|) \mathrm{d} x .
\end{aligned}
$$

Substituting (22) and (23) into (29) shows that

$$
\begin{aligned}
& \frac{\mathrm{d}}{\mathrm{d} t} w_{l}(t) \\
& \quad \geq\left(\int_{\mathbb{R}^{n} \backslash B_{1}}|x|^{\mu} u^{q}(x, t) \psi_{l}(|x|) \mathrm{d} x\right)^{m / q}
\end{aligned}
$$




$$
\begin{aligned}
& \cdot\left(l^{\theta}\left(\int_{\mathbb{R}^{n} \backslash B_{1}}|x|^{\mu} u^{q}(x, t) \psi_{l}(|x|) \mathrm{d} x\right)^{(q-m) / q}-C_{0} C_{1} l^{-2+n+\kappa-m(n+\kappa+\mu) / q}\right) \\
& +\left(\int_{\mathbb{R}^{n} \backslash B_{1}}|x|^{\lambda} \nu^{p}(x, t) \psi_{l}(|x|) \mathrm{d} x\right)^{m / p}\left(\left(\int_{\mathbb{R}^{n} \backslash B_{1}}|x|^{\lambda} \nu^{p}(x, t) \psi_{l}(|x|) \mathrm{d} x\right)^{(p-m) / p}\right. \\
& \left.-C_{0} C_{1} l^{-2+\theta+n+\kappa-m(n+\kappa+\lambda) / p}\right) .
\end{aligned}
$$

Let us discuss the classification of symbols of $A(q, \mu)$ and $A(p, \lambda)$ in (25), (26).

If $A(q, \mu)<0, A(p, \lambda)<0$, we substitute (25) and (26) into (30), and this yields that

$$
\begin{aligned}
& \frac{\mathrm{d} w_{l}(t)}{\mathrm{d} t} \\
& \quad \geq-C_{0} C_{4} l^{m(\theta)} w_{l}^{m}(t)+C_{2} l^{-q(n+\kappa)+n+\kappa+\mu+\theta}\left(\int_{\mathbb{R}^{n} \backslash B_{1}} u(x, t) \psi_{l}(|x|) \mathrm{d} x\right)^{q} \\
& \quad+C_{2} l^{-p(n+\kappa)+n+\kappa+\lambda-p \theta}\left(\int_{\mathbb{R}^{n} \backslash B_{1}} l^{\theta} v(x, t) \psi_{l}(|x|) \mathrm{d} x\right)^{p},
\end{aligned}
$$

where $C_{4}=\max \left\{C_{2}^{m / p}, C_{2}^{m / q}\right\}>0$, and

$$
m(\theta)=\max \{(1-m)(n+\kappa)-2,(1-m)(n+\kappa)-2-(m-1) \theta\}
$$

Set

$$
\theta=\frac{q-p}{p+1}\left(n+\kappa-\frac{\lambda+2}{p-m}\right)
$$

which implies that

$$
-p(n+\kappa)+n+\kappa+\lambda-p \theta=-q(n+\kappa)+n+\kappa+\mu+\theta=\Theta
$$

namely,

$$
\Theta=\frac{\left(-p^{2} q+p q m+p-m\right)(n+\kappa)+(\lambda+2)\left(p q-p^{2}\right)}{(p+1)(p-m)}+\lambda .
$$

By a simple calculation,

$$
\begin{aligned}
& \frac{\mathrm{d} w_{l}(t)}{\mathrm{d} t} \\
& \geq-C_{0} C_{4} l^{m(\theta)} w_{l}^{m}(t) \\
& \quad+C_{2} l^{\Theta}\left(\left(\int_{\mathbb{R}^{n} \backslash B_{1}} u(x, t) \psi_{l}(|x|) \mathrm{d} x\right)^{q}+\left(\int_{\mathbb{R}^{n} \backslash B_{1}} l^{\theta} v(x, t) \psi_{l}(|x|) \mathrm{d} x\right)^{p}\right) \\
& \quad \geq w_{l}^{m}(t)\left(-C_{0} C_{4} l^{m(\theta)}+2^{-(p+q)} C_{2} l^{\Theta} \cdot \min \left\{w_{l}^{p-m}(t), w_{l}^{q-m}(t)\right\}\right) .
\end{aligned}
$$

Note that if $p<p_{c}$, then $m(\theta)<\Theta$. Further, $w_{l}(0)$ is nondecreasing with respect to $l \in$ $(0,+\infty)$ and

$$
\sup \left\{w_{l}(0): l \in(0,+\infty)\right\}>0 \text {. }
$$


Chou et al. Boundary Value Problems

(2021) 2021:36

Page 12 of 20

Then there exists sufficiently large $l_{2}>1$ such that

$$
C_{0} C_{4} l_{2}^{m(\theta)} \leq 2^{-(p+q+1)} C_{2} l_{2}^{\Theta} \cdot \min \left\{w_{l_{2}}^{p-m}(0), w_{l_{2}}^{q-m}(0)\right\} .
$$

Combining (32) with (33), we get

$$
\frac{\mathrm{d} w_{l_{2}}(t)}{\mathrm{d} t} \geq 2^{-(p+q+1)} C_{2} l_{2}^{\Theta} \cdot \min \left\{w_{l_{2}}^{p}(t), w_{l_{2}}^{q}(t)\right\} .
$$

Just like the proof of Theorem 4.1, we can obtain that $(u, v)$ blows up in a finite time.

For $A(q, \mu)=0, A(p, \lambda)<0$, we set $\theta=0$. It follows from (25), (26), and (30) that

$$
\begin{aligned}
& \frac{\mathrm{d} w_{l}(t)}{\mathrm{d} t} \\
& \geq\left(C_{2}(\ln l)^{-(q-1)}\left(\int_{\mathbb{R}^{n} \backslash B_{1}} u(x, t) \psi_{l}(|x|) \mathrm{d} x\right)^{q}\right)^{m / q} \\
& \quad \times\left(C_{2}^{(q-m) / q}(\ln l)^{-(q-1)(q-m) / q}\left(\int_{\mathbb{R}^{n} \backslash B_{1}} u(x, t) \psi_{l}(|x|) \mathrm{d} x\right)^{q-m}\right. \\
& \left.\quad-C_{0} C_{1} l^{n+\kappa-2-m(n+\kappa+\mu) / q}\right) \\
& \quad+\left(C_{2} l^{-p(n+\kappa)+n+\kappa+\lambda}\left(\int_{\mathbb{R}^{n} \backslash B_{1}} v(x, t) \psi_{l}(|x|) \mathrm{d} x\right)^{p}\right)^{m / p} \\
& \quad \times\left(C_{2}^{(p-m) / p} l^{(-p(n+\kappa)+n+\kappa+\lambda)(p-m) / p}\left(\int_{\mathbb{R}^{n} \backslash B_{1}} v(x, t) \psi_{l}(|x|) \mathrm{d} x\right)^{p-m}\right. \\
& \left.\quad-C_{0} C_{1} l^{n+\kappa-2-m(n+\kappa+\lambda) / p}\right) .
\end{aligned}
$$

Here

$$
\begin{aligned}
& n+\kappa-2-m(n+\kappa+\mu) / q<0, \\
& n+\kappa-2-m(n+\kappa+\lambda) / p<(-p(n+\kappa)+n+\kappa+\lambda)(p-m) / p .
\end{aligned}
$$

Then there exists sufficiently large $l_{3}$ such that

$$
\begin{aligned}
& \frac{\mathrm{d} w_{l_{3}}(t)}{\mathrm{d} t} \\
& \geq C_{2}^{m / q}\left(\ln l_{3}\right)^{m(1-q) / q}\left(\int_{\mathbb{R}^{n} \backslash B_{1}} u(x, t) \psi_{l_{3}}(|x|) \mathrm{d} x\right)^{m} \\
& \quad \times \frac{1}{2} C_{2}^{(q-m) / q}\left(\ln l_{3}\right)^{(1-q)(q-m) / q}\left(\int_{\mathbb{R}^{n} \backslash B_{1}} u(x, t) \psi_{l_{3}}(|x|) \mathrm{d} x\right)^{q-m} \\
& \quad+C_{2}^{m / p} l_{3}^{-m(n+\kappa)+m(n+\kappa+\lambda) / p}\left(\int_{\mathbb{R}^{n} \backslash B_{1}} v(x, t) \psi_{l_{3}}(|x|) \mathrm{d} x\right)^{m} \\
& \quad \times \frac{1}{2} C_{2}^{(p-m) / p} l_{3}^{(-p(n+\kappa)+n+\kappa+\lambda)(p-m) / p}\left(\int_{\mathbb{R}^{n} \backslash B_{1}} v(x, t) \psi_{l_{3}}(|x|) \mathrm{d} x\right)^{p-m} \\
& \geq C_{5}\left(\left(\int_{\mathbb{R}^{n} \backslash B_{1}} u(x, t) \psi_{l_{3}}(|x|) \mathrm{d} x\right)^{q}+\left(\int_{\mathbb{R}^{n} \backslash B_{1}} v(x, t) \psi_{l_{3}}(|x|) \mathrm{d} x\right)^{p}\right)
\end{aligned}
$$




$$
\geq 2^{-(p+q)} C_{5} \cdot \min \left\{w_{l_{3}}^{p}(t), w_{l_{3}}^{q}(t)\right\}
$$

where $C_{5}>0$ is a positive constant depending only on $l_{3}$. Therefore, we can obtain that $(u, v)$ blows up in a finite time by a similar proof process of Theorem 4.1.

For other cases, select $\theta=0$. By the similar argument as $A(q, \mu)=0, A(p, \lambda)<0$, we can also prove that any nontrivial solution blows up in a finite time.

Theorem 4.3 Assume that $p, q>m>1, \lambda, \mu>0, \kappa>-n$, and $0 \leq u_{0}, v_{0} \in C_{0}\left(\mathbb{R}^{n} \backslash B_{1}\right)$ are nontrivial. Then, if $p>p_{c}$, there exist both nontrivial global and blow-up solutions to problem (1)-(4).

Proof The comparison principle and Lemma 3.1 can prove the existence of the nontrivial global solution to problem (1)-(4) with sufficiently small initial value. Next, we study the blow-up solution to problem (1)-(4) when the initial value is sufficiently large.

For $l>1$ and $(u, v)$ is the solution to problem (1)-(4), set

$$
\tilde{w}_{l}(t)=\int_{\mathbb{R}^{n} \backslash B_{1}}(u(x, t)+v(x, t)) \psi_{l}(|x|) \mathrm{d} x, \quad t \geq 0 .
$$

According to the Hölder inequality and (30), we have

$$
\begin{aligned}
& \frac{\mathrm{d}}{\mathrm{d} t} \tilde{w}_{l}(t) \\
& \geq \quad\left(\int_{\mathbb{R}^{n} \backslash B_{1}}|x|^{\mu} u^{q}(x, t) \psi_{l}(|x|) \mathrm{d} x\right)^{m / q}\left(\left(\int_{\mathbb{R}^{n} \backslash B_{1}}|x|^{\mu} u^{q}(x, t) \psi_{l}(|x|) \mathrm{d} x\right)^{(q-m) / q}\right. \\
& \left.\quad-C_{0} C_{1} l^{-2+n+\kappa-m(n+\kappa+\mu) / q}\right) \\
& \quad+\left(\int_{\mathbb{R}^{n} \backslash B_{1}}|x|^{\lambda} v^{p}(x, t) \psi_{l}(|x|) \mathrm{d} x\right)^{m / p}\left(\left(\int_{\mathbb{R}^{n} \backslash B_{1}}|x|^{\lambda} v^{p}(x, t) \psi_{l}(|x|) \mathrm{d} x\right)^{(p-m) / p}\right. \\
& \left.\quad-C_{0} C_{1} l^{-2+n+\kappa-m(n+\kappa+\lambda) / p}\right) \\
& \geq \tilde{w}_{l}^{m}(t)\left(-C_{0} C_{1} C_{6}+2^{-(p+q)} C_{7} \cdot \min \left\{\tilde{w}_{l}^{p-m}(t), \tilde{w}_{l}^{q-m}(t)\right\}\right),
\end{aligned}
$$

where

$$
\begin{aligned}
C_{6}= & \max \left\{l^{-2+n+\kappa-m(n+\kappa+\mu) / q}\left(\int_{\mathbb{R}^{n} \backslash B_{1}}|x|^{-\mu /(q-1)} \psi_{l}(|x|) \mathrm{d} x\right)^{(1-q) m / q},\right. \\
& \left.l^{-2+n+\kappa-m(n+\kappa+\lambda) / p}\left(\int_{\mathbb{R}^{n} \backslash B_{1}}|x|^{-\lambda /(p-1)} \psi_{l}(|x|) \mathrm{d} x\right)^{(1-p) m / p}\right\}, \\
C_{7}= & \min \left\{\left(\int_{\mathbb{R}^{n} \backslash B_{1}}|x|^{\mu /(1-q)} \psi_{l}(|x|) \mathrm{d} x\right)^{1-q},\left(\int_{\mathbb{R}^{n} \backslash B_{1}}|x|^{\lambda /(1-p)} \psi_{l}(|x|) \mathrm{d} x\right)^{1-p}\right\} .
\end{aligned}
$$

If $\left(u_{0}, v_{0}\right)$ is so large that

$$
C_{0} C_{1} C_{6} \leq 2^{-(p+q+1)} C_{7} \cdot \min \left\{\tilde{w}_{l}^{p-m}(0), \tilde{w}_{l}^{q-m}(0)\right\},
$$


then (35) leads to

$$
\frac{\mathrm{d} \tilde{w}_{l}(t)}{\mathrm{d} t} \geq 2^{-(p+q+1)} C_{7} \cdot \min \left\{\tilde{w}_{l}^{p}(t), \tilde{w}_{l}^{q}(t)\right\}, \quad t>0 .
$$

By a similar argument in the proof of Theorem 4.1, one can show that $(u, v)$ blows up in a finite time.

\section{The critical case}

In this section, we consider the critical case

$$
p=p_{c}=m+\frac{2+\lambda}{n+\kappa}
$$

Obviously, we can prove that (29), (32) still hold, and

$$
n+\kappa+\mu-q(n+\kappa)=n+\kappa+\lambda-p_{c}(n+\kappa)=(1-m)(n+\kappa)-2 .
$$

The result of the critical case is based on the following three lemmas.

Lemma 5.1 Assume that $(u, v)$ is a nontrivial global solution to problem (1)-(4) with $p=$ $p_{c}$, then there exists $M_{0}>0$ independent of $t$ such that

$$
\int_{\mathbb{R}^{n} \backslash B_{1}}(u(x, t)+v(x, t))|x|^{\kappa} \mathrm{d} x \leq M_{0}, \quad t>0 .
$$

Proof For any sufficiently large $l>1$, it follows from (32) that

$$
\begin{aligned}
& \frac{\mathrm{d} w_{l}(t)}{\mathrm{d} t} \\
& \quad \geq w_{l}^{m}(t) l^{-(m-1)(n+\kappa)-2}\left(-C_{0} C_{4}+2^{-\left(p_{c}+q\right)} C_{2} \cdot \min \left\{w_{l}^{p_{c}-m}(t), w_{l}^{q-m}(t)\right\}\right),
\end{aligned}
$$

where $w_{l}$ is defined by (28) with $\theta=0$. Similar to the end of the proof of Theorem 4.1, there exists some $l_{3}>1$ such that, for any $l>l_{3}$,

$$
2^{-\left(p_{c}+q+1\right)} C_{2} \cdot \min \left\{w_{l}^{p_{c}-m}(t), w_{l}^{q-m}(t)\right\} \leq C_{0} C_{4},
$$

which implies

$$
w_{l}(t) \leq \max \left\{\left(C_{0} C_{4} C_{2}^{-1} 2^{p_{c}+q+1}\right)^{1 /\left(p_{c}-m\right)},\left(C_{0} C_{4} C_{2}^{-1} 2^{p_{c}+q+1}\right)^{1 /(q-m)}\right\} .
$$

Let $l \rightarrow+\infty$ in the above inequality, then we can obtain (38).

Lemma 5.2 Under the assumption of Lemma 5.1, there exist three positive constants $M_{1}, M_{2}, M_{3}>0$ independent of $l$ and $t$ such that, for any sufficiently large $l>1$,

$$
\begin{aligned}
\frac{\mathrm{d} w_{l}(t)}{\mathrm{d} t} \geq & M_{1}^{m-\tau} l^{(1-m)(n+\kappa)-2} w_{l}^{m-\tau}(t) \\
& \times\left(-M_{2}\left(\int_{B_{\delta l} \backslash B_{l}}(u(x, t)+v(x, t)) \psi_{l}(|x|) \mathrm{d} x\right)^{\tau}\right.
\end{aligned}
$$




$$
\left.+M_{1}^{-(m-\tau)} M_{3} \cdot \min \left\{w_{l}^{p_{c}-m+\tau}(t), w_{l}^{q-m+\tau}(t)\right\}\right),
$$

where

$$
0<\tau<\min \left\{\frac{p_{c}-m}{p_{c}-1}, \frac{q-m}{q-1}\right\} .
$$

Proof It is easy to verify that

$$
\begin{aligned}
n+ & \kappa-2-m(n+\kappa+\mu) / q+\tau(\mu-(q-1)(n+\kappa)) / q \\
& =((1-q)(n+\kappa)+\mu)(q-m+\tau) / q, \\
n+ & \kappa-2-m(n+\kappa+\lambda) / p_{c}+\tau\left(\lambda-\left(p_{c}-1\right)(n+\kappa)\right) / p_{c} \\
& =\left(\left(1-p_{c}\right)(n+\kappa)+\lambda\right)\left(p_{c}-m+\tau\right) / p_{c} .
\end{aligned}
$$

For any sufficiently large $l>1$, it follows from the Hölder inequality that

$$
\begin{aligned}
& \int_{B_{\delta l} \backslash B_{l}} u^{m}(x, t) \psi_{l}(|x|) \mathrm{d} x \\
& \leq\left(\int_{B_{\delta l} \backslash B_{l}}|x|^{-(m-\tau) \mu /(q-m-(q-1) \tau)} \psi_{l}(|x|) \mathrm{d} x\right)^{(q-m-(q-1) \tau) / q} \\
& \quad \times\left(\int_{B_{\delta l} \backslash B_{l}}|x|^{\mu} u^{q}(x, t) \psi_{l}(|x|) \mathrm{d} x\right)^{(m-\tau) / q}\left(\int_{B_{\delta l} \backslash B_{l}} u(x, t) \psi_{l}(|x|) \mathrm{d} x\right)^{\tau} \\
& \leq C_{8} l^{n+\kappa-(n+\kappa+\mu) m / q+\tau(\mu-(q-1)(n+\kappa)) / q} \\
& \quad \times\left(\int_{\mathbb{R}^{n} \backslash B_{1}}|x|^{\mu} u^{q}(x, t) \psi_{l}(|x|) \mathrm{d} x\right)^{(m-\tau) / q}\left(\int_{B_{\delta l} \backslash B_{l}} u(x, t) \psi_{l}(|x|) \mathrm{d} x\right)^{\tau}, \\
& \int_{B_{\delta l} \backslash B_{l}} v^{m}(x, t) \psi_{l}(|x|) \mathrm{d} x \\
& \leq\left(\int_{B_{\delta l} \backslash B_{l}}|x|^{-(m-\tau) \lambda /\left(p_{c}-m-\left(p_{c}-1\right) \tau\right)} \psi_{l}(|x|) \mathrm{d} x\right)^{\left(p_{c}-m-\left(p_{c}-1\right) \tau\right) / p_{c}} \\
& \quad \times\left(\int_{B_{\delta l} \backslash B_{l}}|x|^{\lambda} \nu^{p_{c}}(x, t) \psi_{l}(|x|) \mathrm{d} x\right)^{(m-\tau) / p_{c}}\left(\int_{B_{\delta l} \backslash B_{l}} v(x, t) \psi_{l}(|x|) \mathrm{d} x\right)^{\tau} \\
& \leq C_{8} l^{n+\kappa-(n+\kappa+\lambda) m / p_{c}+\tau\left(\lambda-\left(p_{c}-1\right)(n+\kappa)\right) / p_{c}} \\
& \quad \times\left(\int_{\mathbb{R}^{n} \backslash B_{1}}|x|^{\lambda} v^{p_{c}}(x, t) \psi_{l}(|x|) \mathrm{d} x\right)^{(m-\tau) / p_{c}}\left(\int_{B_{\delta l} \backslash B_{l}} v(x, t) \psi_{l}(|x|) \mathrm{d} x\right)^{\tau},
\end{aligned}
$$

where $C_{8}>0$ is a constant independent of $l$. Substituting the above two inequalities into (29) with $\theta=0$, it follows from (25), (26), (37), (40), and (41) that

$$
\begin{aligned}
\frac{\mathrm{d}}{\mathrm{d} t} w_{l}(t) & \\
\geq & -C_{0} C_{8} l^{-(m-1)(n+\kappa)-2}\left(M_{1} w_{l}(t)\right)^{m-\tau}\left(\int_{B_{2 l} \backslash B_{l}}(u(x, t)+v(x, t)) \psi_{l}(|x|) \mathrm{d} x\right)^{\tau} \\
& +2^{-\left(p_{c}+q\right)} C_{2} l^{-(m-1)(n+\kappa)-2} \cdot \min \left\{w_{l}^{p_{c}}(t), w_{l}^{q}(t)\right\},
\end{aligned}
$$


which yields (39) by choosing

$$
M_{1}=\max \left\{C_{2}^{1 / p_{c}}, C_{2}^{1 / q}\right\}, \quad M_{2}=C_{0} C_{8}, \quad M_{3}=2^{-\left(p_{c}+q\right)} C_{2} .
$$

Lemma 5.3 Under the assumption of Lemma 5.1, there exists a constant $M_{4}>0$ independent of $l$ and $t$ such that, for any sufficiently large $l>1$,

$$
\frac{\mathrm{d}}{\mathrm{d} t} \int_{\mathbb{R}^{n} \backslash B_{1}}(u(x, t)+v(x, t)) \psi_{l}(|x|) \mathrm{d} x \geq-M_{4} l^{\left(p_{c}(n+\kappa-2)-m(n+\kappa+\lambda)\right) /\left(p_{c}-m\right)} .
$$

Proof Owing to the Hölder inequality, one obtains

$$
\begin{aligned}
& \int_{B_{2 l} \backslash B_{l}} u^{m}(x, t) \psi_{l}(|x|) \mathrm{d} x \\
& \leq\left(\int_{B_{2 l} \backslash B_{l}}|x|^{-\frac{m \mu}{q-m}} \psi_{l}(|x|) \mathrm{d} x\right)^{(q-m) / q}\left(\int_{B_{2 l} \backslash B_{l}}|x|^{\mu} u^{q}(x, t) \psi_{l}(|x|) \mathrm{d} x\right)^{m / q} \\
& \leq C_{9} l^{n+\kappa-m(n+\kappa+\mu) / q}\left(\int_{B_{2 l} \backslash B_{l}}|x|^{\mu} u^{q}(x, t) \mathrm{d} x\right)^{m / q}, \\
& \int_{B_{2 l} \backslash B_{l}} v^{m}(x, t) \psi_{l}(|x|) \mathrm{d} x \\
& \leq\left(\int_{B_{2 l} \backslash B_{l}}|x|^{-\frac{m \lambda}{p_{c}-m}} \psi_{l}(|x|) \mathrm{d} x\right)^{\left(p_{c}-m\right) / p_{c}}\left(\int_{B_{2 l} \backslash B_{l}}|x|^{\mu} v^{p_{c}}(x, t) \psi_{l}(|x|) \mathrm{d} x\right)^{m / p_{c}} \\
& \leq C_{9} l^{n+\kappa-m(n+\kappa+\lambda) / p_{c}}\left(\int_{B_{2 l} \backslash B_{l}}|x|^{\lambda} v^{p_{c}}(x, t) \mathrm{d} x\right)^{m / p_{c}},
\end{aligned}
$$

where $C_{9}>0$, independent of $l$. Substitute the above results into (29) and

$$
\frac{q(n+\kappa-2)-m(n+\kappa+\mu)}{q-m}=\frac{p_{c}(n+\kappa-2)-m(n+\kappa+\lambda)}{p_{c}-m},
$$

then it follows from the Young inequality that

$$
\begin{aligned}
\frac{\mathrm{d}}{\mathrm{d} t} w_{l}(t) \geq & -C_{0} C_{9} l^{n+\kappa-2-m(n+\kappa+\mu) / q}\left(\int_{B_{2 l} \backslash B_{l}}|x|^{\mu} u^{q}(x, t) \mathrm{d} x\right)^{m / q} \\
& +\int_{\mathbb{R}^{n} \backslash B_{1}}|x|^{\mu} u^{q}(x, t) \psi_{l}(|x|) \mathrm{d} x \\
& -C_{0} C_{9} l^{n+\kappa-2-m(n+\kappa+\lambda) / p_{c}}\left(\int_{B_{2 l} \backslash B_{l}}|x|^{\lambda} \nu^{p_{c}}(x, t) \mathrm{d} x\right)^{m / p_{c}} \\
& +\int_{\mathbb{R}^{n} \backslash B_{1}}|x|^{\lambda} v^{p}(x, t) \psi_{l}(|x|) \mathrm{d} x \\
\geq & -\frac{m}{q} \int_{\mathbb{R}^{n} \backslash B_{1}}|x|^{\mu} u^{q}(x, t) \psi_{l}(|x|) \mathrm{d} x+\int_{\mathbb{R}^{n} \backslash B_{1}}|x|^{\mu} u^{q}(x, t) \psi_{l}(|x|) \mathrm{d} x \\
& -\frac{q-m}{q}\left(C_{0} C_{9}\right)^{q /(q-m)} l^{(q(n+\kappa-2)-m(n+\kappa+\mu)) /(q-m)} \\
& -\frac{m}{p_{c}} \int_{\mathbb{R}^{n} \backslash B_{1}}|x|^{\lambda} \nu^{p}(x, t) \psi_{l}(|x|) \mathrm{d} x+\int_{\mathbb{R}^{n} \backslash B_{1}}|x|^{\lambda} \nu^{p}(x, t) \psi_{l}(|x|) \mathrm{d} x
\end{aligned}
$$




$$
\begin{aligned}
& -\frac{p_{c}-m}{p_{c}}\left(C_{0} C_{9}\right)^{p_{c} /\left(p_{c}-m\right)} l^{\left(p_{c}(n+\kappa-2)-m(n+\kappa+\lambda)\right) /\left(p_{c}-m\right)} \\
\geq & -M_{4} l^{\left(p_{c}(n+\kappa-2)-m(n+\kappa+\lambda)\right) /\left(p_{c}-m\right)}
\end{aligned}
$$

where

$$
M_{4}=\max \left\{\frac{q-m}{q}\left(C_{0} C_{9}\right)^{q /(q-m)}, \frac{p_{c}-m}{p_{c}}\left(C_{0} C_{9}\right)^{p_{c} /\left(p_{c}-m\right)}\right\} .
$$

Now we prove the following theorem.

Theorem 5.1 Assume that $\kappa>-n$. Then any nontrivial solution to problem (1)-(4) with $p=p_{c}$ blows up in a finite time.

Proof We prove the theorem by contradiction. Assume that $(u, v)$ is a nontrivial global solution to problem (1)-(4) with $p=p_{c}$. Set

$$
\Lambda=\sup _{l>0, t>0} w_{l}(t)=\sup _{t>0} \int_{\mathbb{R}^{n} \backslash B_{1}}(u(x, t)+v(x, t))|x|^{\kappa} \mathrm{d} x .
$$

It follows from (38) and the nontriviality of $(u, v)$ that $0<\Lambda<+\infty$. Owing to (43) and the monotonicity of $w_{l}(t)$ with respect to $l \in(0,+\infty)$, there exist $l_{0}>1$ and $t_{0}>0$ such that, for any $0<\varepsilon<\Lambda$,

$$
w_{l_{0} / \delta}\left(t_{0}\right) \geq \Lambda-\varepsilon
$$

From Lemma 5.3, for $s \geq t_{0}$, we obtain

$$
\begin{aligned}
\int_{\mathbb{R}^{n} \backslash B_{1}}(u(x, s)+v(x, s)) \psi_{l_{0} / \delta}(|x|) \mathrm{d} x \\
\geq \int_{\mathbb{R}^{n} \backslash B_{1}}\left(u\left(x, t_{0}\right)+v\left(x, t_{0}\right)\right) \psi_{l_{0} / \delta}(|x|) \mathrm{d} x \\
\quad-M_{4}\left(l_{0} / \delta\right)^{\left(p_{c}(n+\kappa-2)-m(n+\kappa+\lambda)\right) /\left(p_{c}-m\right)}\left(s-t_{0}\right) \\
\geq \\
\geq \Lambda-\varepsilon-M_{4}\left(l_{0} / \delta\right)^{\left(p_{c}(n+\kappa-2)-m(n+\kappa+\lambda)\right) /\left(p_{c}-m\right)}\left(s-t_{0}\right),
\end{aligned}
$$

which yields that

$$
\begin{aligned}
& \int_{B_{\delta l_{0}} \backslash B_{l_{0}}}(u(x, s)+v(x, s)) \psi_{l_{0}}(|x|) \mathrm{d} x \\
& \quad \leq \int_{\mathbb{R}^{n} \backslash B_{1}}(u(x, t)+v(x, t))|x|^{\kappa} \mathrm{d} x-\int_{\mathbb{R}^{n} \backslash B_{1}}(u(x, s)+v(x, s)) \psi_{l_{0} / \delta}(|x|) \mathrm{d} x \\
& \quad \leq \varepsilon+M_{4}\left(l_{0} / \delta\right)^{\left(p_{c}(n+\kappa-2)-m(n+\kappa+\lambda)\right) /\left(p_{c}-m\right)}\left(s-t_{0}\right), \quad s \geq t_{0} .
\end{aligned}
$$

Let $l=l_{0}$ in (39), from the above inequality, one gets that

$$
\frac{\mathrm{d} w_{l_{0}}(t)}{\mathrm{d} t} \geq M_{1}^{m-\tau} l_{0}^{(1-m)(n+\kappa)-2} w_{l_{0}}^{m-\tau}(t)
$$




$$
\begin{aligned}
& \times\left(-M_{2}\left(\int_{B_{\delta l_{0}} \backslash B_{l_{0}}}(u(x, t)+v(x, t)) \psi_{l_{0}} \mathrm{~d} x\right)^{\tau}\right. \\
& \left.+M_{1}^{-(m-\tau)} M_{3} \cdot \min \left\{w_{l_{0}}^{p_{c}-m+\tau}(t), w_{l_{0}}^{q-m+\tau}(t)\right\}\right) \\
\geq & M_{1}^{m-\tau} l_{0}^{(1-m)(n+\kappa)-2} w_{l_{0}}^{m-\tau}(t) \\
& \times\left(-M_{2}\left(\varepsilon+M_{4}\left(l_{0} / \delta\right)^{\left(p_{c}(n+\kappa-2)-m(n+\kappa+\lambda)\right) /\left(p_{c}-m\right)}\left(s-t_{0}\right)\right)^{\tau}\right. \\
& \left.+M_{1}^{-(m-\tau)} M_{3} \cdot \min \left\{w_{l_{0}}^{p_{c}-m+\tau}(t), w_{l_{0}}^{q-m+\tau}(t)\right\}\right) .
\end{aligned}
$$

Take $\varepsilon_{0} \in(0, \Lambda)$ and $M_{5}>0$ to get

$$
M_{2}\left(\varepsilon_{0}+M_{5}\right)^{\tau} \leq \frac{1}{2} M_{1}^{-(m-\tau)} M_{3} \cdot \min \left\{(\Lambda-\varepsilon)^{p_{c}-m+\tau}(t),(\Lambda-\varepsilon)^{q-m+\tau}(t)\right\},
$$

where $\varepsilon_{0}$ and $M_{5}$ are independent of $l_{0}, 0<\tau<\min \left\{\frac{p_{c}-m}{p_{c}-1}, \frac{q-m}{q-1}\right\}$. Then we obtain

$$
\frac{\mathrm{d} w_{l_{0}}(t)}{\mathrm{d} t} \geq \frac{1}{2} M_{3} l_{0}^{(1-m)(n+\kappa)-2} \cdot \min \left\{w_{l_{0}}^{p_{c}}(t), w_{l_{0}}^{q}(t)\right\}, \quad t_{0}<t<t_{1},
$$

where

$$
t_{1}=t_{0}+\frac{M_{5}}{M_{4}}\left(l_{0} / \delta\right)^{\left(-p_{c}(n+\kappa-2)+m(n+\kappa+\lambda)\right) /\left(p_{c}-m\right)} .
$$

Integrating (44) over $\left(t_{0}, t_{1}\right)$ with respect to $t$ and using

$$
\left(p_{c}(n+\kappa-2)-m(n+\kappa+\lambda)\right) /\left(p_{c}-m\right)=(1-m)(n+\kappa)-2,
$$

one gets that

$$
\begin{aligned}
w_{l_{0}}\left(t_{1}\right) & \\
\geq & w_{l_{0}}\left(t_{0}\right)+\frac{1}{2} M_{3} l_{0}^{(1-m)(n+\kappa)-2} \cdot \min \left\{\left(\Lambda-\varepsilon_{0}\right)^{p_{c}},\left(\Lambda-\varepsilon_{0}\right)^{q}\right\}\left(t_{1}-t_{0}\right) \\
\geq & w_{l_{0} / \delta}\left(t_{0}\right)+\frac{1}{2} M_{3} l_{0}^{(1-m)(n+\kappa)-2} \cdot \min \left\{\left(\Lambda-\varepsilon_{0}\right)^{p_{c}},\left(\Lambda-\varepsilon_{0}\right)^{q}\right\} \\
& \times \frac{M_{5}}{M_{4}}\left(l_{0} / \delta\right)^{\left(-p_{c}(n+\kappa-2)+m(n+\kappa+\lambda)\right) /\left(p_{c}-m\right)} \\
= & w_{l_{0} / \delta}\left(t_{0}\right) \\
& +\frac{M_{3} M_{5}}{2 M_{4}} \delta^{\left(p_{c}(n+\kappa-2)-m(n+\kappa+\lambda)\right) /\left(p_{c}-m\right)} \cdot \min \left\{\left(\Lambda-\varepsilon_{0}\right)^{p_{c}},\left(\Lambda-\varepsilon_{0}\right)^{q}\right\} .
\end{aligned}
$$

That is to say,

$$
\int_{\mathbb{R}^{n} \backslash B_{1}}\left(u\left(x, t_{1}\right)+v\left(x, t_{1}\right)\right)|x|^{\kappa} \mathrm{d} x \geq w_{l_{0}}\left(t_{1}\right) \geq w_{l_{0} / \delta}\left(t_{0}\right)+\gamma_{0} \geq \Lambda-\varepsilon_{0}+\gamma_{0},
$$

where

$$
\gamma_{0}=\frac{M_{3} M_{5}}{2 M_{4}} \delta^{\left(p_{c}(n+\kappa-2)-m(n+\kappa+\lambda)\right) /\left(p_{c}-m\right)} \cdot \min \left\{\left(\Lambda-\varepsilon_{0}\right)^{p_{c}},\left(\Lambda-\varepsilon_{0}\right)^{q}\right\}
$$


is a positive constant independent of $l_{0}$. It is obviously verified that

$$
w_{\left(\delta l_{0}\right) / \delta}\left(t_{1}\right)=w_{l_{0}}\left(t_{1}\right) \geq \Lambda-\varepsilon_{0}+\gamma_{0} \geq \Lambda-\varepsilon_{0} .
$$

Using the same method, one gets

$$
\int_{\mathbb{R}^{n} \backslash B_{1}}\left(u\left(x, t_{2}\right)+v\left(x, t_{2}\right)\right)|x|^{\kappa} \mathrm{d} x \geq w_{\delta l_{0}}\left(t_{2}\right) \geq w_{l_{0}}\left(t_{1}\right)+\gamma_{0} \geq \Lambda-\varepsilon_{0}+2 \gamma_{0},
$$

where

$$
t_{2}=t_{1}+\frac{M_{5}}{M_{4}} l_{0}^{\left(-p_{c}(n+\kappa-2)+m(n+\kappa+\lambda)\right) /\left(p_{c}-m\right)} .
$$

Similarly, for any positive integer $i$, we obtain

$$
\begin{aligned}
& \int_{\mathbb{R}^{n} \backslash B_{1}}\left(u\left(x, t_{i}\right)+v\left(x, t_{i}\right)\right)|x|^{\kappa} \mathrm{d} x \\
& \quad \geq w_{\delta^{i-1} l_{0}}\left(t_{i}\right) \geq w_{\delta^{i-2} l_{0}}\left(t_{i-1}\right)+\delta_{0} \geq \Lambda-\varepsilon_{0}+i \gamma_{0},
\end{aligned}
$$

where

$$
t_{i}=t_{i-1}+\frac{M_{5}}{M_{4}}\left(\delta^{i-2} l_{0}\right)^{-\left(p_{c}(n+\kappa-2)+m(n+\kappa+\lambda)\right) /\left(p_{c}-m\right)} .
$$

Letting $i \rightarrow+\infty$ in (45) implies

$$
\sup _{t>0} \int_{\mathbb{R}^{n} \backslash B_{1}}(u(x, t)+v(x, t))|x|^{\kappa} \mathrm{d} x=+\infty
$$

which contradicts (38).

\section{Acknowledgements}

The authors would like to thank the referees for their valuable comments and suggestions which improved the original manuscript.

\section{Funding}

This work is supported by the National Natural Science Foundation of China (No. 11871133), by the Department of Science and Technology of Jilin Province (YDZJ202101ZYTS044).

\section{Availability of data and materials}

Not applicable.

\section{Competing interests}

The authors declare that they have no competing interests.

\section{Authors' contributions}

All the authors contributed to each part of this study equally and approved the final version of the manuscript.

\section{Author details}

${ }^{1}$ School of Mathematics, Jilin University, 130012 Changchun, China. ${ }^{2}$ School of Science, Northeast Electric Power University, 132012 Jilin, China.

\section{Publisher's Note}

Springer Nature remains neutral with regard to jurisdictional claims in published maps and institutional affiliations. 


\section{References}

1. Aguirre, J., Escobedo, M.: On the blow-up of solutions of a convective reaction diffusion equation. Proc. R. Soc. Edinb., Sect. A 123(3), 433-460 (1993)

2. Deng, K., Levine, H.: The role of critical exponents in blow-up theorems: the sequel. J. Math. Anal. Appl. 243, 85-126 (2000)

3. Díaz, J.I., Hernández, J., Ilyasov, Y.Sh.: On the exact multiplicity of stable ground states of non-Lipschitz semilinear elliptic equations for some classes of starshaped sets. Adv. Nonlinear Anal. 9(1), 1046-1065 (2020)

4. Escobedo, M., Herrero, M.: Boundedness and blow up for a semilinear reaction-diffusion system. J. Differ. Equ. 89, 176-202 (1991)

5. Fujita, $H_{\text {.: }}$ On the blowing up of solutions of the Cauchy problem for $u_{t}=\Delta u+u^{1+\alpha}$. J. Fac. Sci., Univ. Tokyo, Sect. I 13 , 109-124 (1966)

6. Galaktionov, V.: Blow-up for quasilinear heat equations with critical Fujita's exponents. Proc. R. Soc. Edinb. A 124, 517-525 (1994)

7. Galaktionov, V., Kurdjumov, S., Mikhailov, A., Samarskii, A.: On unbounded solutions of the Cauchy problem for the parabolic equation $\partial u / \partial t=\nabla(u \sigma \nabla u)+u^{\beta}$. Dokl. Akad. Nauk SSSR 252, 1362-1364 (1980)

8. Ghoul, T.-E., Nguyen, V.T., Zaag, H.: Construction of type I blowup solutions for a higher order semilinear parabolic equation. Adv. Nonlinear Anal. 9(1), 388-412 (2020)

9. Guo, W., Gao, Y.: Critical Fujita curves for a class of coupled convection-diffusion systems. J. Jilin Univ. Sci. Ed. 58(2), $271-276(2020)$

10. Guo, W., Lei, M.: Critical Fujita curves for a coupled reaction-convection-diffusion system with singular coefficients. J. Jilin Univ. Sci. Ed. 54, 183-188 (2016)

11. Guo, W., Wang, X., Zhou, M.: Asymptotic behavior of solutions to a class of semilinear parabolic equations. Bound. Value Probl. 2016, 68, 1-9 (2016)

12. Hayakawa, K.: On nonexistence of global solutions of some semilinear parabolic equations. Proc. Jpn. Acad. 49 503-525 (1973)

13. Kobayashi, K., Siaro, T., Tanaka, H.: On the blowing up problem for semilinear heat equations. J. Math. Soc. Jpn. 29, 407-424 (1977)

14. Leng, Y., Nie, Y., Zhou, Q.: Asymptotic behavior of solutions to a class of coupled nonlinear parabolic systems. Bound Value Probl. 2019, 68, 1-11 (2019)

15. Levine, H.: The role of critical exponents in blowup theorems. SIAM Rev. 32(2), 262-288 (1990)

16. Li, F., Lin, H., Liu, B.: Blow-up analyses in reaction-diffusion equations with Fujita exponents. Nonlinear Anal., Real World Appl. 50, 386-412 (2019)

17. Li, H., Wang, X., Nie, Y., He, H.: Asymptotic behavior of solutions to a degenerate quasilinear parabolic equation with a gradient term. Electron. J. Differ. Equ. 2015, 295, 1-12 (2015)

18. Lian, W., Wang, J., Xu, R.: Global existence and blow up of solutions for pseudo-parabolic equation with singular potential. J. Differ. Equ. 269(6), 4914-4959 (2020)

19. Liu, C., Wang, H.: Global existence and nonexistence of solutions for Newtonian filtration equation. Appl. Anal. 96(8), 1379-1389 (2017)

20. Martynenko, A., Tedeev, A.: Cauchy problem for a quasilinear parabolic equation with a source term and an inhomogeneous density. Comput. Math. Math. Phys. 47(2), 238-248 (2007)

21. Na, Y., Zhou, M., Zhou, X., Gai, G.: Blow-up theorems of Fujita type for a semilinear parabolic equation with a gradient term. Adv. Differ. Equ. 2018, 128, 1-12 (2018)

22. Qi, Y., Levine, H.: The critical exponent of degenerate parabolic systems. Z. Angew. Math. Phys. 44, $249-265$ (1993)

23. Quittner, P., Souplet, P.: Superlinear Parabolic Problems. Blow-up, Global Existence and Steady States. Birkhäuser Advanced Texts: Basler Lehrbücher. Birkhäuser, Basel (2007)

24. Rault, J.: The Fujita phenomenon in exterior domains under dynamical boundary conditions. Asymptot. Anal. 66(1), $1-8(2010)$

25. Wang, C., Zheng, S.: Critical Fujita exponents of degenerate and singular parabolic equations. Proc. R. Soc. Edinb., Sect. A 136(2), 415-430 (2006)

26. Wang, C., Zheng, S., Wang, Z.: Critical Fujita exponents of a class of quasilinear equations with homogeneous Neumann boundary data. Nonlinearity 20, 1343-1359 (2007)

27. Weissler, F.: Existence and non-existence of global solutions for semilinear equation. Isr. J. Math. 6(1), 85-126 (2000)

28. Wu, Z., Zhao, J., Yin, J., Li, H.: Nonlinear Diffusion Equations. World Scientific, River Edge (2001)

29. Zheng, S., Wang, C.: Large time behaviour of solutions to a class of quasilinear parabolic equations with convection terms. Nonlinearity 21(9), 2179-2200 (2008)

30. Zhou, M., Li, H., Guo, W., Zhou, X.: Critical Fujita exponents to a class of non-Newtonian filtration equations with fast diffusion. Bound. Value Probl. 2016, 146, 1-16 (2016)

31. Zhou, Q., Nie, Y., Han, X.: Large time behavior of solutions to semilinear parabolic equations with gradient. J. Dyn. Control Syst. 22(1), 191-205 (2016) 